\title{
Qualidade física e sensorial da carne de cordeiros de três genótipos alimentados com rações formuladas com duas relações volumoso:concentrado
}

\author{
Roberto Germano Costa ${ }^{1}$, Neube Michel dos Santos ${ }^{2}$, Wandrick Hauss de Sousa ${ }^{3}$, Rita de \\ Cássia Ramos do Egypto Queiroga ${ }^{1}$, Paulo Sérgio de Azevedo ${ }^{1}$, Felipe Queiroga Cartaxo ${ }^{3}$
}

\footnotetext{
${ }^{1}$ Universidade Federal da Paraíba - UFPB, Programa de Doutorado Integrado em Zootecnia - PDIZ.

2 Agência Estadual de Vigilância Sanitária - AGEVISA/PB.

${ }^{3}$ Empresa Estadual de Pesquisa Agropecuária - EMEPA/PB.
}

RESUMO - Objetivou-se avaliar os atributos sensoriais (dureza, odor, sabor, suculência e cor) e físicos (força de cisalhamento, perda por cocção e parâmetros de cor $\mathrm{L}^{*}, \mathrm{a}^{*}, \mathrm{~b}^{*}$ ), além do conteúdo de ferro da carne de cordeiros dos grupos Santa Inês (SI), Dorper $\times$ Santa Inês $(D P \times S I)$ e Sem Padrão Racial Definido (SRD) terminados em confinamento com dietas formuladas com duas relações volumoso:concentrado (50:50 e 20:80). Foram utilizados 54 animais (18 de cada genótipo, com peso médio inicial de $23 \mathrm{~kg}$ e 150 dias de idade) abatidos com $36 \mathrm{~kg}$ de peso vivo. As dietas aplicadas favoreceram a diferenciação na cor da carne. A intensidade de brilho $\left(\mathrm{L}^{*}\right)$ diferiu entre os grupos genéticos. Nos animais terminados com a dieta com alto nível de concentrado, a carne apresentou menores perdas por cocção. Observou-se a existência de correlação entre a intensidade de vermelho $\left(\mathrm{a}^{*}\right)$ e a cor da carne in natura e entre a concentração de ferro dietético na carne e a intensidade de brilho $\left(\mathrm{L}^{*}\right)$. A carne de cordeiros SI e SRD possui menor dureza e maior suculência à carne de cordeiros Dorper $\times$ Santa Inês. A relação volumoso:concentrado na dieta não promove modificações nos atributos sensoriais e parâmetros físicos da carne.

Palavras-chave: cor, dureza, perda por cocção, sabor, suculência

\section{Physical and sensorial quality of lamb meat of three different genotypes fed diets formulated with two roughage:concentrate ratios}

\begin{abstract}
The objective of this work was to evaluate sensory atributes (tenderness, odor, flavor, juiciness and color) and physical attributes (shearing force, cooking losses and the color parameters of $\mathrm{L}^{*}, \mathrm{a}^{*}, \mathrm{~b}^{*}$ ) in addition to content of iron in the meat of Santa Inês (SI), Dorper $\times$ Santa Inês $(\mathrm{DP} \times \mathrm{SI})$ and Without Definite Breed Pattern (WDB) lambs, finished in feedlots, in function of two roughage:concentrate (50:50 and 20:80) relationships in the diet. It was used 54 whole lambs (18 from each genotype, with initial average weight of $23 \mathrm{~kg}$ and at 150 days of age) slaughtered at $36 \mathrm{~kg}$ of live weight. The fed diets favored differentiation in meat color. Bright intensity $\left(\mathrm{L}^{*}\right)$ differed among the genetic groups. In animals fed diet with high level of concentrate, the meat presented fewer cooking losses. It was observed the existence of a correlation between the intensity of red $\left(\mathrm{a}^{*}\right)$ and the color of fresh meat and among dietary iron concentration in the meat and and bright intensity $\left(\mathrm{L}^{*}\right)$. Meat of SI and SRD lambs is less tough and more juicy than meat of Dorper $\times$ Santa Inês. The ratio roughage:concentrate in the diet does not promote changes in sensory attributes and physical parameters of the meat.
\end{abstract}

Key Words: color, cooking losses, flavor, juiciness, toughness

\section{Introdução}

O mercado, potencialmente promissor para carne ovina no Brasil, aliado ao novo perfil dos consumidores, mais perceptíveis à qualidade, tem induzido o aprimoramento técnico e organizacional na atual cadeia produtiva. O desenvolvimento de técnicas adequadas de manejo nutricional para cordeiros em terminação que conduzam a modificações desejáveis no conteúdo dos ácidos graxos da carne tem promovido, em alguns casos, a depreciação nos atributos sensoriais da carne, a exemplo do aroma e sabor (Vasta \& Priolo, 2006). Neste contexto, a utilização de níveis adequados de volumoso na dieta de cordeiros em terminação, pode promover modificações desejáveis no perfil dos ácidos graxos da carne (Gallo et al., 2007) e alterações na qualidade sensorial da carne. Essas informações permitirão viabilizar a diferenciação dos produtos e desenvolver estratégias específicas para cada 
empreendimento produtivo, otimizando os processos de comercialização.

Embora a avaliação das características sensoriais da carne ovina promova resultados geralmente inquestionáveis sobre os diversos fatores que determinam as tendências de consumo (Martínez-Cerezo et al., 2005a), trabalhos contendo esta metodologia ainda não são amplamente utilizados. A análise sensorial representa uma importante ferramenta de avaliação da qualidade da carne, contudo, a aplicação simultânea com técnicas instrumentais pode especificar com maior eficácia a aceitação do produto no mercado (MartínezCerezo et al., 2005b). A cor desempenha importante papel na qualidade sensorial da carne e destaca-se como principal fator de apreciação no momento da compra. Normalmente, a coloração da carne é determinada pela concentração total de mioglobina (proteína envolvida nos processos de oxigenação do músculo) e pelas proporções relativas desse pigmento no tecido muscular, que pode ser encontrado na forma de mioglobina reduzida, com coloração púrpura, oximioglobina, de cor vermelho brilhante e metamioglobina, normalmente marrom(Renerre, 1990).

Entre os fatores pré-abate que podem promover alterações na qualidade sensorial da carne ovina, destacase o genótipo animal (Martínez-Cerezo et al., 2005b). Neste sentido, a caracterização dos atributos sensoriais da carne de cordeiros Santa Inês, de mestiços procedentes de cruzamentos terminais como o Dorper $\times$ Santa Inês e de animais sem padrão racial definido ou SRD, parcela significativa do efetivo ovino presente na Região Nordeste do país, pode possibilitar a ampliação na demanda desses produtos por meio de desenvolvimento de estratégias adequadas de marketing.

Objetivou-se avaliar os atributos sensoriais (dureza, odor, sabor, suculência e cor) e físicos (força de cisalhamento, perda por cocção e os parâmetros de cor $L^{*}$, a ${ }^{*}, b^{*}$ ) da carne de cordeiros Santa Inês, Dorper × Santa Inês e SRD alimentados com dietas formuladas com duas relações volumoso:concentrado.

\section{Material e Métodos}

A pesquisa foi conduzida na Estação Experimental de Pendência, pertencente à Empresa Estadual de Pesquisa Agropecuária da Paraíba (EMEPA), localizada no município de Soledade, microrregião do Cariri Paraibano.

Foram utilizados 54 cordeiros inteiros, 18 da raça Santa Inês, $18 \mathrm{~F}_{1}$ Dorper $\times$ Santa Inês e $18 \mathrm{SRD}$, com peso médio inicial de 23 kg e média de 150 dias de idade, distribuídos individualmente em gaiolas, medindo $0,80 \times 1,20 \mathrm{~m}$, providas de comedouros e bebedouros. O período de adaptação consistiu de 14 dias e as pesagens dos cordeiros foram efetuadas semanalmente para o controle do ganho de peso.

Os animais foram inicialmente vacinados contra clostridioses e vermifugados com aplicação subcutânea de ivermectina a $1 \%$.

As dietas isoproteicas (Tabelas 1 e 2), fornecidas duas vezes ao dia, foram calculadas para atender às exigências em proteína bruta e energia metabolizável, de modo a permitir aos animais ganhos de $250 \mathrm{~g} / \mathrm{dia}$, de acordo com as recomendações contidas no NRC (2007). Foi preestabelecido consumo de 5\% de matéria seca em relação ao peso vivo, ajustando-se conforme sobra do dia anterior, de acordo com metodologia utilizada por Cartaxo (2009). As dietas consistiram de duas relações volumoso:concentrado, correspondentes a 50:50 e 20:80.

Os cordeiros foram abatidos, após jejum de sólidos de 18 horas, com 36 kg de peso vivo ou, quando não atingiam este peso em 63 dias de confinamento, eram abatidos. O abate foi realizado em concordância com as normas vigentes do Regulamento de Inspeção Industrial e Sanitária dos Produtos de Origem Animal (Brasil, 1997). As carcaças foram penduradas pelos tendões em ganchos apropriados, distanciadas 17 cm uma da outra e resfriadas em câmara frigorífica a temperatura de $4 \pm 1^{\circ} \mathrm{C}$, por 24 horas, todas envoltas em sacos plásticos transparentes, para reduzir a perda de umidade superficial, comum no processo de resfriamento. Após o período de refrigeração, foram

Tabela 1 - Composição química dos ingredientes das dietas (\% MS)

\begin{tabular}{|c|c|c|c|c|c|c|c|c|}
\hline Ingrediente & $\begin{array}{c}\text { Matéria } \\
\text { seca } \\
(\%)\end{array}$ & $\begin{array}{c}\text { Proteína } \\
\text { bruta } \\
(\%)\end{array}$ & $\begin{array}{l}\text { Extrato } \\
\text { etéreo } \\
(\%)\end{array}$ & $\begin{array}{c}\text { Energia } \\
\text { metabolizável* }^{*}\end{array}$ & $\begin{array}{l}\text { Nutrientes } \\
\text { digestíveis } \\
\text { totais (\%) }\end{array}$ & $\begin{array}{c}\text { Fibra em } \\
\text { detergente } \\
\text { neutro }(\%)\end{array}$ & $\begin{array}{c}\text { Carboidratos } \\
\text { não-fibrosos } \\
(\%)\end{array}$ & $\begin{array}{c}\text { Matéria } \\
\text { mineral } \\
(\%)\end{array}$ \\
\hline Feno de tifton & 88,96 & 8,96 & 1,63 & 2,01 & 55,59 & 78,78 & 7,44 & 6,72 \\
\hline Milho moído & 87,64 & 9,11 & 4,07 & 3,15 & 87,12 & 13,98 & 74,47 & 1,55 \\
\hline Farelo de trigo & 89,00 & 16,63 & 3,53 & 2,61 & 72,19 & 44,30 & 33,93 & 5,58 \\
\hline Óleo de soja & 99,55 & - & 99,04 & 7,48 & 207,27 & - & - & - \\
\hline
\end{tabular}

* Mcal/kg MS - NRC (1985) 
lumborum, entre a $4^{0}$ e 5ํㅡ vértebras lombares, evitando-se dentro do possível, o contato com gordura e tecido conectivo (Cezar \& Sousa, 2007). Para a realização das análises de ferro, amostras do músculo longissimus lumborum foram descongeladas à temperatura de $4 \pm 1{ }^{\circ} \mathrm{C}$, por 24 horas. Em seguida, as amostras foram trituradas em liquidificador doméstico, marca Arno, por um período médio de 5 minutos, até completa homogeneização do material para determinação de cinzas, conforme metodologia descrita na AOAC (2000), método 920.153. Os teores de ferro foram obtidos seguindo método colorimétrico, descrito por Ranganna (1991), em espectrofotômetro UV/visível (Coleman SP/295, São Paulo), com comprimento de onda de $480 \mathrm{~nm}$.

O delineamento experimental utilizado foi inteiramente casualizado, em esquema fatorial $3 \times 2$ (três genótipos e duas dietas), com nove repetições para as análises de cor $\left(\mathrm{L}^{*}, \mathrm{a}^{*}, \mathrm{~b}^{*}\right)$, perda por cocção, força de cisalhamento e ferro, e três repetições por provador para a análise sensorial. Procedeu-se à interpretação estatística dos resultados por meio de análise de variância e desenvolveu-se correlação de Pearson entre as características físicas (PPC, FC, $\mathrm{L}^{*}$, a ${ }^{*}$, $\mathrm{b}^{*}$ ) e os atributos sensoriais (suculência, dureza, cor in natura e cor cozida) e entre os parâmetros de cor $\left(\mathrm{L}^{*}\right.$, a ${ }^{*} \mathrm{e}$ $\mathrm{b}^{*}$ ) e o teor de ferro da carne. O modelo estatístico utilizado foi: $Y_{i j k}=\mu+G_{i}+D_{j}+G D_{i j}+\varepsilon_{i j k}$, em que: $Y_{i j k}=$ valor observado da variável dependente estudada; $\mu=$ média geral; $G_{i}=$ efeito do genótipo i; $D_{j}=$ efeito da dieta j; $G D_{i j}=$ interação genótipo $\times$ dieta; $\varepsilon_{i j k}=$ erro aleatório associado a cada observação. As médias foram comparadas pelo teste Tukey a 5\% de probabilidade, utilizando-se o PROC GML do programa SAS (2001).

\section{Resultados e Discussão}

O genótipo não influenciou o peso final, o ganho de peso médio diário e o período de confinamento (Tabela 4). A dieta com 20:80 de volumoso:concentrado favoreceu a obtenção de índices produtivos mais satisfatórios (ganho de peso e período de confinamento).
Segundo Furusho-Garcia et al. (2004), o cruzamento de ovinos Santa Inês com raças especializadas para corte tem sido preconizado como importante ferramenta para elevar a produção de carne e melhorar a eficiência produtiva. De acordo com Barros et al. (2005), a terminação de cordeiros Dorper $\times$ Santa Inês em confinamento caracterizou-se como prática de manejo produtivo economicamente viável na Região Nordeste do País, mas neste trabalho os cordeiros deste grupo genético não se sobressaíram $(\mathrm{P}>0,05)$, em termos de ganho de peso, em relação aos animais dos demais grupos pesquisados.

A dieta com alto nível de concentrado, e consequentemente com maior $(\mathrm{P}<0,05)$ valor energético, favoreceu o acúmulo mais acentuado de gordura na carcaça (Tabela 4). Os cordeiros Dorper $\times$ Santa Inês apresentaram maior quantidade de gordura na carcaça, seguido dos SRD e dos Santa Inês, com menor percentual, resultado possivelmente decorrente da maior precocidade dos mestiços de Dorper (Madruga et al., 2006).

As relações volumoso:concentrado favoreceram a diferenciação na cor da carne in natura (Tabela 5). A coloração menos intensa (menos vermelha e mais amarela) observada na carne dos cordeiros terminados com a dieta com relação 20:80 de volumoso:concentrado, possivelmente decorreu da maior concentração de pigmentos carotenoides presentes nos lipídios da carne, que determinou tonalidade de amarelo mais acentuada (Bressan et al., 2004).

As relações volumoso:concentrado na dieta dos cordeiros não influenciaram os parâmetros de odor, dureza, suculência e sabor. O genótipo, no entanto, interferiu na suculência e na dureza da carne, frequentemente o principal parâmetro qualitativo utilizado para avaliar a satisfação do consumidor (Martínez-Cerezo et al., 2005a). A carne dos ovinos Santa Inês e SRD destacou-se com menor dureza em relação à dos cordeiros DP $\times$ SI, bem como pela maior suculência em relação ao meio sangue. De acordo com Sañudo et al. (1998), possivelmente maiores concentrações de gordura intramuscular favorecem a obtenção de carnes mais macias. Segundo Siqueira et al.(2001)e Pardi et al.(2001),

Tabela 4 - Desempenho de cordeiros em confinamento recebendo dietas formuladas com duas relações volumoso:concentrado na dieta

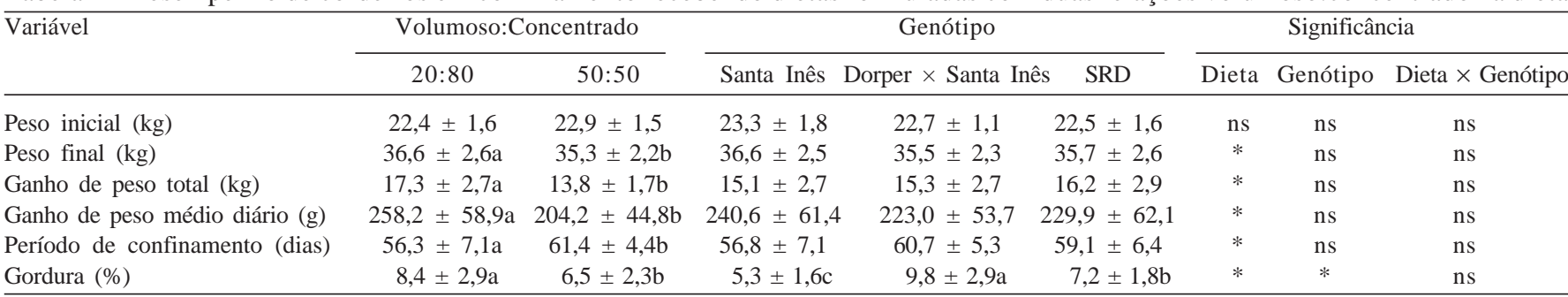

a,b - Médias seguidas por letras distintas diferem entre si pelo teste Tukey.

ns, valores não-significativos $(\mathrm{P}>0,05) ;{ }^{*} \mathrm{P}<0,05$. 
a gordura subcutânea e inter e intramuscular pode ter influência direta na dureza da carne, ao atuarem como isolante térmico, evitando o resfriamento rápido da carcaça e o encurtamento dos sarcômeros, acarretando maior dureza da carne.

A perda de peso por cocção (PPC) caracteriza-se como importante parâmetro de avaliação da qualidade da carne. Associa-se ao rendimento no preparo para o consumo e influencia a suculência da carne e, neste estudo, diferiu entre os genótipos avaliados, com menores valores na carne dos cordeiros SRD em relação aos obtidos na carne dos ovinos Santa Inês (Tabela 6). A dieta com menor percentual de volumoso proporcionou menores perdas por cocção na carne. De acordo com Sañudo et al. (1997), maiores níveis de gordura intra (marmoreio) e intermuscular conduzem a menores perdas de peso por cocção e, consequentemente, a obtenção de carnes mais suculentas, visto que a gordura presente na carne atua como barreira contra a perda de umidade. Entretanto, Pardi et al. (2001) referenciaram que maiores PPC na carne ovina decorriam de maiores quantidades de gordura presente nos tecidos, possivelmente, porque além da umidade, parte da gordura da carne é perdida com o processamento térmico.

Com base nos resultados desta pesquisa, pode-se considerar que a utilização de reprodutores Dorper em sistemas de cruzamento terminais com fêmeas da raça Santa Inês na produção de cordeiros Dorper $\times$ Santa Inês não proporciona a obtenção de carnes com melhor qualidade sensorial.

Além de métodos sensoriais, a dureza da carne também pode ser determinada por técnicas instrumentais. Bickerstffe et al. (1997), avaliando cortes comerciais de bovinos, ovinos e suínos, classificaram a carne ovina como muito macia, quando apresentasse força de cisalhamento de até $8 \mathrm{Kgf} / \mathrm{cm}^{2}$, aceitável com valores variando de 8 e $11 \mathrm{Kgf} / \mathrm{cm}^{2}$ e dura acima de $11 \mathrm{Kgf} / \mathrm{cm}^{2}$ e, considerando que nesta pesquisa foi utilizado a mesma metodologia aplicada por estes autores, pode-se classificar as carnes de cordeiros avaliados (4,9 a 5,4 Kgf $/ \mathrm{cm}^{2}$ ) como macias.

A força de cisalhamento mensurada em lâmina de Warner-Bratzler geralmente tem apresentado alta correlação com a análise sensorial (Otremba et al., 1999). Devine et al. (1993) observaram relação linear entre a dureza da carne obtida por teste sensorial e a força de cisalhamento, com coeficiente de regressão de 0,7 , resultado não constatado nesta pesquisa, cujos valores comprovaram baixos coeficientes de correlação $(\mathrm{P}>0,05)$.

O pH final da carne em torno de 5,5 (Tabela 6), próximo ao ponto isoelétrico das proteínas e, de acordo com Watanabe et al. (1996), com ausência da força de atração existente entre as fibras musculares, proporcionadas pela diferença das cargas positivas e negativas, favoreceu o distanciamento entre os miofilamentos que constituem o tecido muscular, aumentando o comprimento dos

Tabela 5 - Atributos sensoriais da carne de cordeiros terminados em confinamento com dietas formuladas com duas relações volumoso:concentrado

\begin{tabular}{|c|c|c|c|c|c|c|c|c|c|}
\hline \multirow[t]{2}{*}{ Variável } & \multicolumn{2}{|c|}{ Volumoso:Concentrado } & \multicolumn{3}{|c|}{ Genótipo } & \multicolumn{4}{|c|}{ Significância } \\
\hline & $20: 80$ & $50: 50$ & Santa Inês & Dorper $\times$ Santa Inês & SRD & Dieta & Genótipo & Dieta & $\times$ Genótipo \\
\hline Cor in natura & $4,14 \pm 1,6 b$ & $4,97 \pm 1,8 a$ & $4,69 \pm 1,9$ & $4,60 \pm 1,4$ & $4,38 \pm 1,9$ & $* *$ & ns & & ns \\
\hline Odor & $4,85 \pm 1,1$ & $4,63 \pm 1,2$ & $4,73 \pm 1,2$ & $4,76 \pm 1,0$ & $4,73 \pm 1,2$ & ns & ns & & ns \\
\hline Dureza & $2,85 \pm 1,6$ & $2,90 \pm 1,8$ & $2,64 \pm 1,8 b$ & $3,60 \pm 1,7 a$ & $2,39 \pm 1,4 b$ & ns & $* *$ & & ns \\
\hline Suculência & $4,50 \pm 1,4$ & $4,43 \pm 1,5$ & $4,65 \pm 1,4 a$ & $4,04 \pm 1,3 b$ & $4,69 \pm 1,4 a$ & ns & $*$ & & ns \\
\hline Sabor & $4,70 \pm 1,5$ & $4,74 \pm 1,4$ & $4,69 \pm 1,4$ & $4,80 \pm 1,5$ & $4,68 \pm 1,4$ & ns & ns & & ns \\
\hline
\end{tabular}

a,b - Médias seguidas por letras distintas diferem entre si pelo teste Tukey.

$\mathrm{ns}$, valores não-significativos $(\mathrm{P}>0,05) ;{ }^{*} \mathrm{P}<0,05 ;{ }^{* *} \mathrm{P}<0,01$.

Tabela 6 - Parâmetros físico-químicos da carne de cordeiros em função da relação volumoso:concentrado na dieta

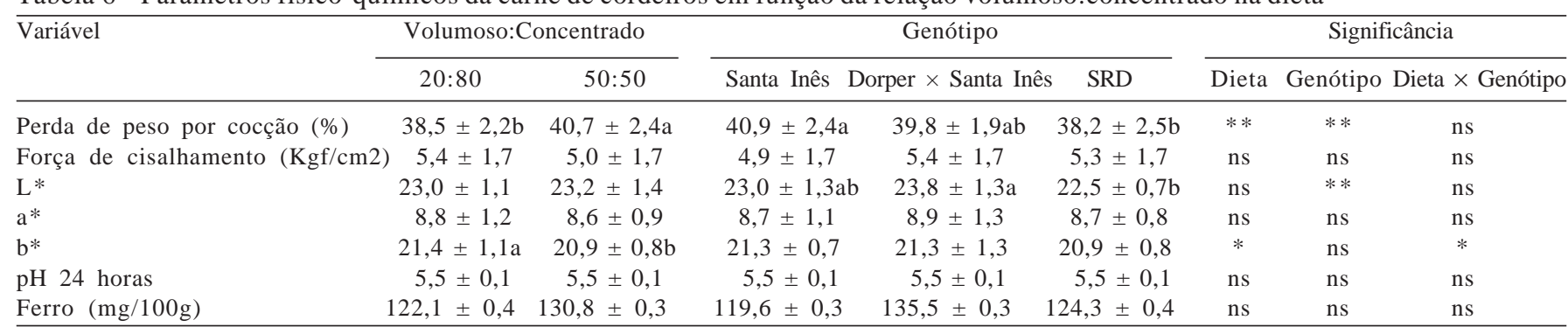

$\mathrm{L}^{*}, \mathrm{a}^{*}$ e $\mathrm{b}^{*}$, correspondem à intensidade de brilho, de vermelho e de amarelo na carne, respectivamente.

a,b - Médias seguidas por letras distintas diferem entre si pelo teste Tukey.

ns, valores não-significativos $(\mathrm{P}>0,05) ;{ }^{*} \mathrm{P}<0,05 ;{ }^{* *} \mathrm{P}<0,01$. 
sarcômeros e conduzindo a menor dureza da carne. É possível que a maior solubilidade do colágeno, e consequentemente a menor concentração de ligações cruzadas intermoleculares (termoestáveis), tenha favorecido a menor dureza observada na carne dos cordeiros avaliados (Sañudo et al., 1996).

A intensidade de brilho na carne $\left(\mathrm{L}^{*}\right)$ diferiu entre os grupos genéticos $(\mathrm{P}>0,05)$ e possivelmente foi influenciada pela deposição de pigmentos no tecido muscular e adiposo ((Dhanda et al., 2003). Os resultados observados para esta variável foram inferiores aos constatados por Bressan et al. (2001), Silva Sobrinho et al. (2005) e Martínez-Cerezo et al. (2005c), em virtude da baixa concentração de pigmentos hemínicos, comprovadapelobaixo conteúdo de ferronas amostras pesquisadas, resultados semelhantes aos constatados nesta pesquisa foram obtidos por Costa et al. (2009).

A intensidade de vermelho na carne ( $\mathrm{a}^{*}$ ) não diferiu entre os genótipos avaliados nem entre as dietas fornecidas, presumindo-se que os níveis de mioglobina na carne dos cordeiros não diferiram. Essa justificativa pode ser confirmada pela ausência de variações nos teores de ferro das amostras de carne analisadas. Contudo, os valores absolutos para esta variável foram inferiores aos referenciados na literatura (Martínez-Cerezo et al., 2005c) e possivelmente foram proporcionados pelo sistema de produção adotado, onde animais em confinamento são menos susceptíveis ao desenvolvimento de atividades físicas, como observado em sistemas extensivos, o que induz a menor síntese de mioglobina, tendo em vista a menor necessidade de oxigenação do músculo, favorecendo a coloração menos intensa na carne (Vestergaard et al., 2000). Houve correlação entre $\mathrm{a}^{*} \mathrm{e}$ a cor da carne in natura dos cordeiros $(0,27)$. Após o desdobramento da interação constatada na intensidade de amarelo $\left(\mathrm{b}^{*}\right)$, observaram-se maiores valores na carne dos cordeiros Santa Inês alimentados com a dieta com maior nível de concentrado, possivelmente decorrentes de maiores concentrações de lipídios intra e intermuscular e, portanto, de quantidades superiores de pigmentos carotenóides no tecido adiposo da carne (Bressan et al., 2004). A diferença $(\mathrm{P}<0,05)$ no conteúdo de lipídios (Tabela 4) da carcaça dos cordeiros provavelmente favoreceu a diferenciação $(\mathrm{P}<0,05)$ observada no parâmetro $\mathrm{b}^{*}$ entre estes genótipos, visto que, de acordo com Bressan et al. (2004), pigmentos carotenoides presentes no tecido adiposo, intra e intermuscular, são os principais responsáveis pela tonalidade amarela observada na carne. Além da concentração lipídica, a ingestão de pigmentos carotenoides contidos na fração volumosa verde pelo ruminante pode influenciar a intensidade de amarelo da carne e, consequentemente, o parâmetro b* (Fernandes et al., 2008).

\section{Conclusões}

A carne de cordeiros Santa Inês e Sem Padrão Racial Definido terminados em confinamento tem menor dureza e maior suculência em comparação à de cordeiros mestiços Dorper × Santa Inês. A relação volumoso:concentrado da dieta não promove modificações significativas nos atributos sensoriais e parâmetros físicos da carne de cordeiros terminados em confinamento.

\section{Referências}

AOAC. Official methods of analysis of AOAC International 19.ed. Washington, D.C., 2000, p.1219.

BARROS, N.N.; VASCONCELOS, V.R.; WANDER, A.E. et al Eficiência bioeconômica de cordeiros $\mathrm{F}_{1}$ Dorper $\times$ Santa Inês para produção de carne. Pesquisa Agropecuária Brasileira v.40, n.8, p.825-831, 2005.

BICKERSTAFFE, R.; LE COUTEUR, C.E.; MORTON, J.D. Consistency of tenderness in New Zealand retail meat. In: International Congress of Meat Science Technology, v.43, p.196-197, 1997.

BRASIL. MINISTÉRIO DA AGRICULTURA. Regulamento da inspeção industrial e sanitária de produtos de origem animal. Brasília: MA, 1997. 154p.

BRESSAN, M.C.; JARDIM, N.S.; PEREZ, J.R.O. et al. Influência do sexo e faixas de peso ao abate nas características físicoquímicas da carne de capivara. Ciência e Tecnologia de Alimentos, v.24, n.3, p.357-362, 2004.

BRESSAN, M.C.; PRADO, O.V.; PÉREZ, J.R.O. et al. Efeito do peso ao abate de cordeiros Santa Inês e Bergamácia sobre as características físico-químicas da carne. Ciência Tecnologia de Alimentos, v.21, n.3, p.293-303, 2001.

CARTAXO, F.Q. Desempenho e características da carcaça de cordeiros de diferentes genótipos submetidos a duas dietas. 2009. 89f. Tese (Doutorado em Zootecnia) - Centro de Ciências Agrárias/ Universidade Federal da Paraíba, Areia

CEZAR, M.F.; SOUSA, W.H. Carcaças ovinas e caprinas: obtenção, avaliação e classificação. Uberaba, MG: Ed. Agropecuária Tropical, 2007. 231p.

COSTA, R.C.; BATISTA, A.S.M.; AZEVEDO, P.S et al. Lipid profile of lamb meat from different genotypes submitted to diets with different energy levels. Revista Brasileira de Zootecnia, v.38, n.3, p.532-538, 2009.

DEVINE, C.E.; GRAAFHUIS, A.E.; MUIR, P.D. et al. The effect of growth rate and ultimate $\mathrm{pH}$ on meat quality of lambs. Meat Science, v.35, n.1, p.63-77, 1993.

DHANDA, J.S.; TAYLOR, D.G.; MURRAY, P.J. Growth, carcass and meat quality parameters of male goats: effects of genotype and live weight at slaughter. Small Ruminant Research, v.50, p.57-66, 2003

DUCKETT, S.K.; KLEIN, T.A.; LECKIE, R.K. et al. Effect of freezing on calpastatin activity and tenderness of callipyge lamb. Journal of Animal Science, v.76, n.7, p.1869-1874, 1998

FERNANDES, A.R.M.; SAMPAIO, A.A.M.; HENRIQUE, W. et al. Características da carcaça e da carne de bovinos sob diferentes dietas, em confinamento. Arquivos Brasileiros de Medicina Veterinária e Zootecnia, v.60, n.1, p.139-147, 2008.

FURUSHO-GARCIA, I.F.; PEREZ, J.R.O.; BONAGURIO, S. et al. Desempenho de cordeiros Santa Inês puros e cruzas Santa Inês com Texel, Ile de France e Bergamácia. Revista Brasileira de Zootecnia, v.33, n.6, p.1591-1603, 2004.

GALLO, S.B.; SIQUEIRA, E.R.; ROSA, G.T. Efeito da nutrição da ovelha e do cordeiro sobre o perfil de ácidos graxos do músculo 
Triceps brachii de cordeiros. Revista Brasileira de Zootecnia, v.36, n.6, p.2069-2073, 2007 (supl.).

MAcFIE, H.J.; BRATCHEL, N.; GREENHOFF, K. et al. Design to balance the effect of order of apresentation and frist-order carry-order effects in hall tests. Journal of Sensory Studies, v.4, p.129-148, 1989.

MADRUGA, M.S.; ARAÚJO, W.O.; SOUSA, W.H. et al. Efeito do genótipo e do sexo sobre a composição química e o perfil de ácidos graxos da carne de cordeiros. Revista Brasileira de Zootecnia, v.35, n.4, p.1838-1844, 2006 (supl.).

MARTÍNEZ-CEREZO, S.; SAÑUDO, C.; PANEA, B. et al. Breed, slaughter weight and ageing time effects on consumer appraisal of three muscles of lamb. Meat Science, v.69, p.797-805, 2005a.

MARTÍNEZ-CEREZO, S.; SAÑUDO, C.; MEDEL, I. et al. Breed, slaughter weight and ageing time effects on sensory characteristics of lamb. Meat Science, v.69, p.571-578, 2005b.

MARTÍNEZ-CEREZO, S.; SAÑUDO, C.; PANEA, B. et al. Breed, slaughter weight and ageing time effects on physico-chemical characteristics of lamb meat. Meat Science, v.69, p.325-333, 2005c.

NATIONAL RESEARCH COUNCIL - NRC. Nutrients requirements of small ruminants. 1.ed. Washington, D.C.: National Academy Press, 2007. 362p.

OTREMBA, M.M.; DiKEMAN, M.E.; MILIKEN, G.A. et al. Interrelationships among evaluations of beef longissimus and semitendinosus muscle tenderness by Warner-Bratzler shear force, a descriptive texture profile sensory panel, and a descriptive attribute sensory panel. Journal of Animal Science, v.77, n.4, p.865-873, 1999.

PARDI, M.C.; SANTOS, I.F.; SOUZA, E.R. et al. Ciência, higiene e tecnologia da carne. 2.ed., v.1. Goiânia: Centro Editorial e Gráfico da Universidade Federal de Goiás, 2001. 623p.

RANGANNA, S. Handbook of analysis and quality control for fruits and vegetable products. New Delhi: Mcgraw-Hill, 1991. 1112p.
RENERRE, M. Review: factors involved in the discoloration of beef meat. Journal Food Science Technology, v.25, p.613-630, 1990.

SAÑUDO, C.; NUTE, G.R.; CAMPO, M.M. et al. Assessment of commercial lamb meat quality by British and Spanish taste panels. Meat Science, v.48, p.91-100, 1998.

SAÑUDO, C.; CAMPO, M.M.; SIERRA, I. et al. Breed effect on carcass and meat quality of suckling lambs. Meat Science, v.46, n.4, p.357-365, 1997.

SAÑUDO, C.; SANTOLARIA, M.P.; MARÍA G. et al. Influence of carcass weight on instrumental and sensory lamb meat quality in intensive production systems. Meat Science, v.42, n.2, p.195-202, 1996.

SILVA SOBRINHO, A.G.; PURCHAS, R.W.; KADIM, I.T. et al. Características de qualidade da carne de ovinos de diferentes genótipos e idades ao abate. Revista Brasileira de Zootecnia, v.34, n.3, p.1070-1078, 2005.

SIQUEIRA, E.R.; SIMÕES, C.D.; FERNANDES, S. Efeito do sexo e do peso ao abate sobre a produção de carne de cordeiros. Morfometria da carcaça, peso dos cortes, composição tecidual e componentes não constituintes da carcaça. Revista Brasileira de Zootecnia, v.30, n.4, p.1299-1307, 2001.

STATISTICAL ANALYSIS SYSTEM - SAS. SAS user's guide: statistics. 5.ed. Cary: 2001. 956p.

STONE, H.; SIDEL, J.; OLIVER, S. et al. Sensory evaluation by quantitative descriptive analysis. Food Technology, v.28, n.11, p.24-34, 1974.

VASTA, V.; PRIOLO, A. Ruminant fat volatiles as affected by diet. A review. Meat Science, v.73, p.218-228, 2006.

VESTERGAARD, M.; OKSBJERG, N.; HENCKEL, P. Influence of feeding intensity, grazing and finishing feeding on muscle fibre characteristics and meat colour of semitendinosus, longissimus dorsi and supraspinatus muscle of young bulls. Meat Science, v.54, p.177-185, 2000.

WATANABE, A.; DALY, C.C.; DEVINE, C.E. The effect of the ultimate $\mathrm{pH}$ of meat on tenderness changes during ageing. Meat Science, v.42, p.67-78, 1996. 